\title{
Development of Real-Time Electromyography Controlled 3D Printed Robot Hand Prototype
}

Preliminary Communication

\author{
Mohamad Aizat Abdul Wahit \\ Universiti Putra Malaysia, Department of Electrical and Electronics Engineering, \\ Serdang, Selangor, Malaysia \\ mohamadaizat.mppupm1516@gmail.com
}

\section{Fatimahtul Zahrah Romzi}

Universiti Putra Malaysia, Department of Electrical and Electronics Engineering, Serdang, Selangor, Malaysia

fatimahtulzahrahromzi@gmail.com

\author{
Siti Anom Ahmad \\ Universiti Putra Malaysia, Malaysian Research Institute on Ageing, \\ Serdang, Selangor, Malaysia \\ sanom@upm.edu.my
}

\section{Mohd Hamiruce Marhaban}

Universiti Putra Malaysia, Department of Electrical and Electronics Engineering, Serdang, Selangor, Malaysia

mhm@upm.edu.my

\section{Wada Chikamune}

Kyushu Institute of Technology, Department of Human Intelligence Systems, Graduate School of Life Science and Systems Engineering, Japan wada@brain.kyutech.ac.jp

\begin{abstract}
Developing an anthropomorphic robotic hand (ARH) has become a relevant research field due to the need to help the amputees live their life as normal people. However, the current state of research is unsatisfactory, especially in terms of structural design and the robot control method. This paper, which proposes a 3D printed ARH structure that follows the average size of an adult human hand, consists of five fingers with a tendon-driven actuator mechanism embedded in each finger structure. Besides that, the movement capability of the developed 3D printed robot hand validated by using motion capture analysis to ensure the similarity to the expected motion range in structural design is achieved. Its system functionality test was conducted in three stages: (1) muscular activity detection, (2) object detection for individual finger movement control, and (3) integration of both stages in one algorithm. Finally, an ARH was developed, which resembles human hand features, as well as a reliable system that can perform opened hand palm and some grasping postures for daily use.
\end{abstract}

Keywords - anthropomorphic robotic hand (ARH), electromyography (EMG), reliable system, robot hand.

\section{INTRODUCTION}

In 2012, about 350,000 out of the total of 29.5 million Malaysian population were registered as disabled, and more than 100,000 people were categorized as upper limb amputees and paralyzed with physical disabilities [1]. The fact shows how vital research is related to the development of a robot hand device as a way to help these people perform their daily activities as a normal human does. The anthropomorphic robot hand is one of the robot hand device classes which resembles the human hand. Thus, it emphasizes the reliability and aesthetic value of the device [2]. The device normally is less complex and able to perform a simple task such as opened hand palm and basic grasping postures. A hand prosthesis device should be capable of operating full day that may include the energy harvesting mechanism [3-8] to limit the number of battery charging cycles [9].

In recent decades, robot hand control has undergone evolution, and the conventional joystick controller is not the only interface that can be used as a robot control interface. There are many alternatives to the robot 
control interface available such as an electromyography (EMG) sensor and many more. An EMG sensor is used to provide muscular activity information as inputs for the system. It becomes the most frequently used robot control interface due to its transparent characteristic, which allows the user to control the robot as their body part $[10,11]$. Besides, it is a non-invasive and easy-to-use sensor. In an effort to support the growth of the robot hand research field, 3D printed technology has become the best fabrication method option for this application, as recommended in Industrial Revolution 4.0. It triggers a massive revolution in robot hand production to produce printable prosthetic hand design which is customizable to the wearer and can be printed easily by anyone and anywhere virtually [12]

Recently, some of the researchers have produced robots that have incomplete fingers that do not resemble the actual anatomy of the human hand. They applied the cable-driven actuator mechanism into structural design [13-18]. We found that the mechanism negatively affects motion accuracy of the joint due to the elastic characteristic of the material itself $[19,20]$. Besides, several kinds of research came out with five fingers and the tendon-driven actuator mechanism, but finger movement does not actuate individually [21], and it is bigger than the average size of an adult human hand [22]. We also found that robot structure made of metal [23] is heavy and difficult to be fabricated compared to a 3D printed product in terms of cutting and shaping the product. Currently, 3D printed technology has gained attention of researchers working on a prosthesis hand [14, 16, 18, 24], and it became the fabrication method most frequently used for this purpose.

This paper describes robot hand structural design that follows the average size of an adult human hand and fabricates it by means of 3D printing technology as a recommended fabrication method in the previous study. We hypothesized that the 3D printed robot structure with a tendon-driven actuator mechanism could achieve optimum similarity to the expected motion range as in structural design. For robot control, integration of both an EMG sensor and infra-red sensors into the system allows five fingers to move individually.

\section{METHODOLOGY}

Fig. 1 shows an overview of the project; this research started with two streams simultaneously categorized as structure and system development. In terms of structure development, it consists of a review of current research on structural design of the robot hand and a list of features applied to structural design as improvement. Robot structural design was visualized in a three-dimensional view. Next, finalized structure design was fabricated by using 3D printing technology. In terms of system development, circuit design of the system was constructed, and the electronic hardware components were assembled. Then, the electronic components were combined with the $3 \mathrm{D}$ printed robot structure to complete a prototype. The movement capability of the printed product was examined through motion analysis by using motion capture equipment. Finally, the prototype will be tested for its functionality and reliability to perform several hand postures.

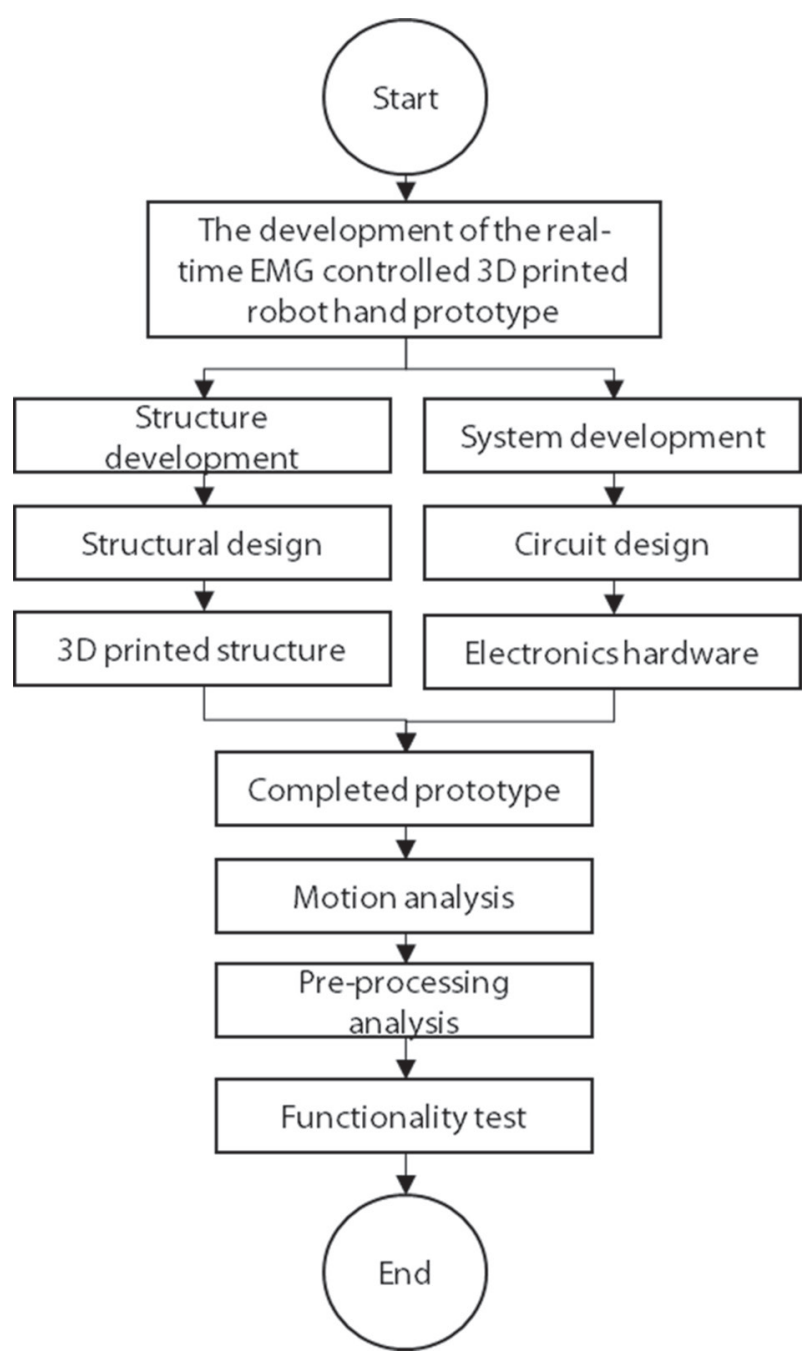

Fig. 1. Project overview.

\subsection{STRUCTURAL DESIGN}

This section shows the features of robot hand structural design to improve robot hand structural design of the previous study. The design is illustrated in threedimensional (3D) drawing using computer-aided software called Inventor Professional 2018 (Autodesk). The features applied in the design are listed below:

- $\quad$ Five fingers with individual movement. Each human hand consists of five fingers that move independently of each other. The fingers are known as the thumb, index, middle, ring and baby fingers. Each finger has three bones known as distal phalanx (DP), intermediate phalanx (IP) and proximal phalanx (PP), with the exception of the thumb that has only DP and PP. In the middle of two bones there are joints known as distal interphalangeal (DIP), proximal interphalangeal (PIP) 
and metacarpophalangeal (MCP). These joint locations are shown in Fig. 2. The number degree of freedom (DOF) of the robot hand is the same as the number of joints involved in structural design, and it is about 14 DOFs. Each finger is allocated one actuator.

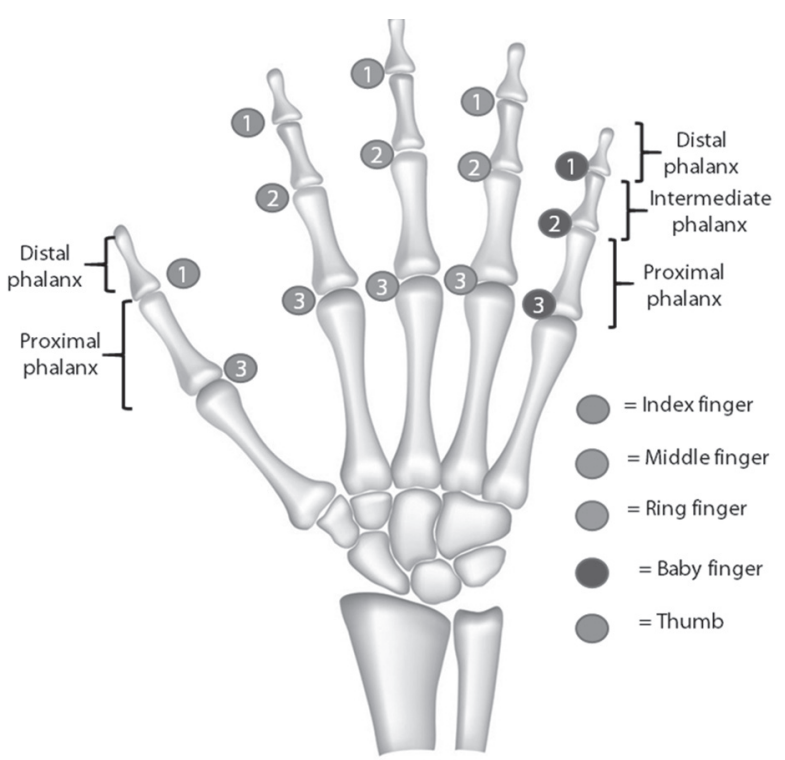

Fig. 2. Human skeletal hand structure:

$1=$ Distal interphalangeal,

$2=$ Proximal interphalangeal,

3= Metacarpophalangeal.

- The planar rigid body motion limits on the sagittal plane only, as shown in Fig. 3.

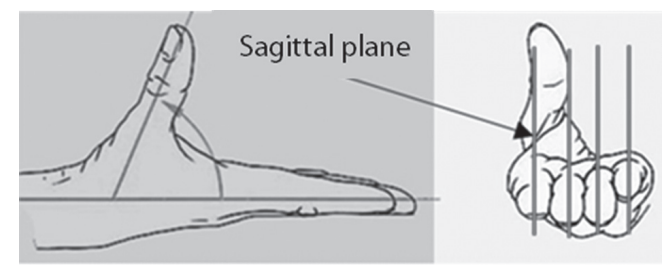

Fig. 3. Sagittal plane of the planar rigid body.

- Tendon-driven actuator mechanism, as shown in Fig. 4. This mechanism uses a solid connector that connects the actuator and robot structure to improve the motion range accuracy of the current robot hand structural design with a cabledriven actuator mechanism. The extension and flexion motion of the finger are shown in Fig. 4(a) and $4(d)$, respectively.

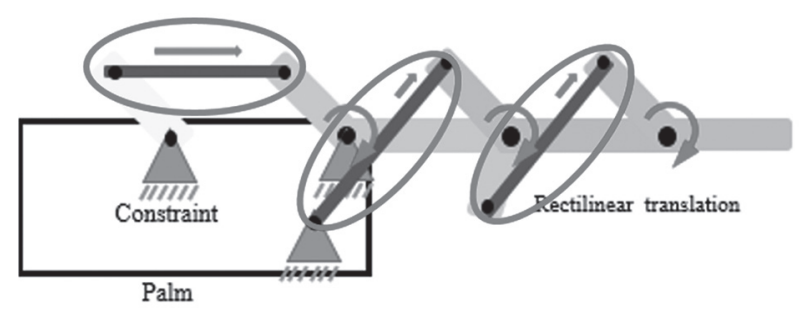

(a)

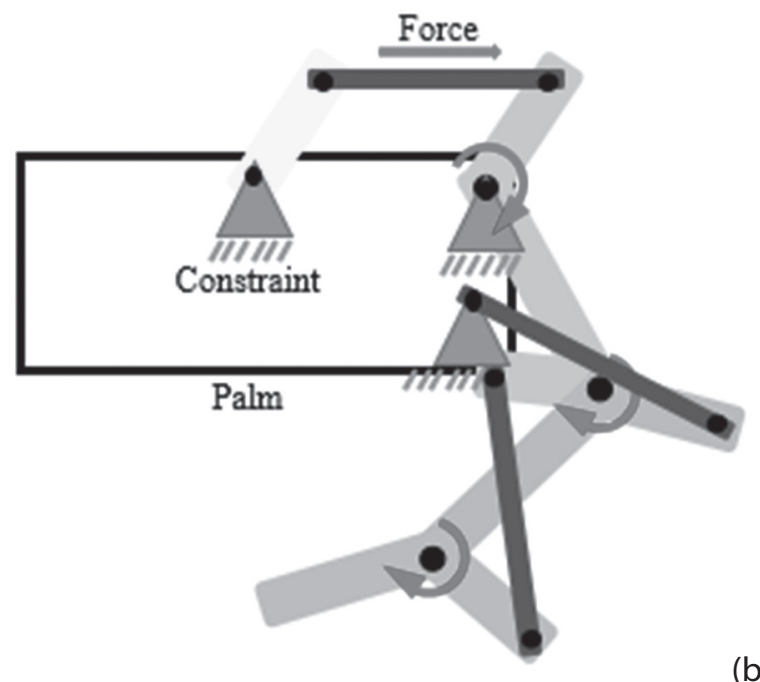

Fig. 4. Robot structure with a tendon-drive actuator: (a) extension motion, (b) flexion motion.

- The thumb tilted $45^{\circ}$ to the left and normal to the hand palm, as shown in Fig. 5. This feature is used to improve the handgrip of the grasping posture.

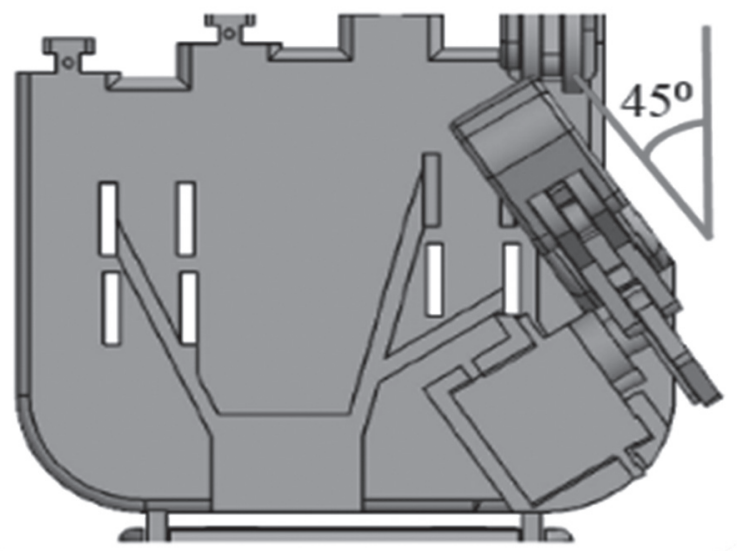

Fig. 5. The thumb of the robot hand tilted $45^{\circ}$ to the left.

- Follow the average size of the adult human hand as an effort to make a robot that resembles an actual human hand [20]. The details of the finger bone lengths are shown in Table 1.

Table 1. The lengths of finger bones

\begin{tabular}{ccc}
$\begin{array}{c}\text { Index, Middle, Ring and Baby Fingers } \\
\text { Lame of bone }\end{array}$ & $\begin{array}{c}\text { Thumb } \\
\text { Length }(\mathbf{m})\end{array}$ & $\begin{array}{c}\text { Length }(\mathbf{m}) \\
\text { DP }\end{array}$ \\
IP & 0.02325 & 0.02325 \\
PP & 0.02400 & - \\
\hline
\end{tabular}

- Limits the motion range of the joints by applying the mechanical stopper to design, as shown in Fig. 6. The details of the motion range of the joint are shown in Table 2. 


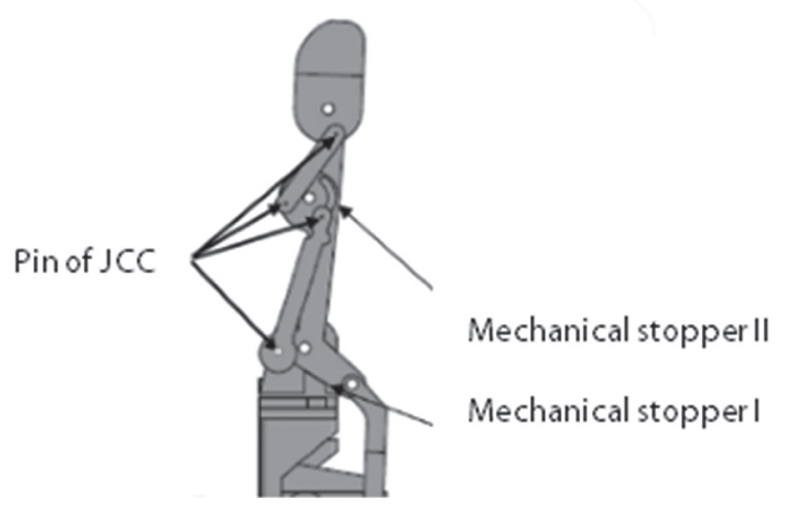

Fig. 6. Mechanical stopper of the finger structure.

Table 2. Mechanical stopper of the finger structure.

\begin{tabular}{|c|c|c|}
\hline \multirow{2}{*}{ Joints } & \multicolumn{2}{|r|}{ Fingers } \\
\hline & Thumb & Index, Middle, Ring, Baby \\
\hline DIP & $85^{\circ}$ & $90^{\circ}$ \\
\hline PIP & - & $105^{\circ}$ \\
\hline MCP & $90^{\circ}$ & $85^{\circ}$ \\
\hline
\end{tabular}

\subsection{CIRCUIT DESIGN AND ELECTRONICS HARDWARE}

By referring to the block diagram shown in Fig. 7 and the electronic hardware configuration of the system shown in Fig. 8, there are two detections involved in the system known as muscular activity and object detection. For muscular activity detection, it was performed by using the Myoware EMG sensor, while object detection was performed by using four units of S-LFS-4-4 ways IR sensors. These sensors act as the input interface of the processor (the Arduino Uno microcontroller). The microcontroller used to process the input signal and control the (TowerPro SG90) servo motor (five units). The movements are produced because the tendon mechanism connects the actuator to the robot structure. The signals can be monitored by connecting the microcontroller to the laptop through a universal serial bus port. The power source of the system is supplied from the power grid $(240 \mathrm{~V} 60 \mathrm{~Hz})$, and it is converted into DC power supply (5V 2A) by using a DC-DC converter.

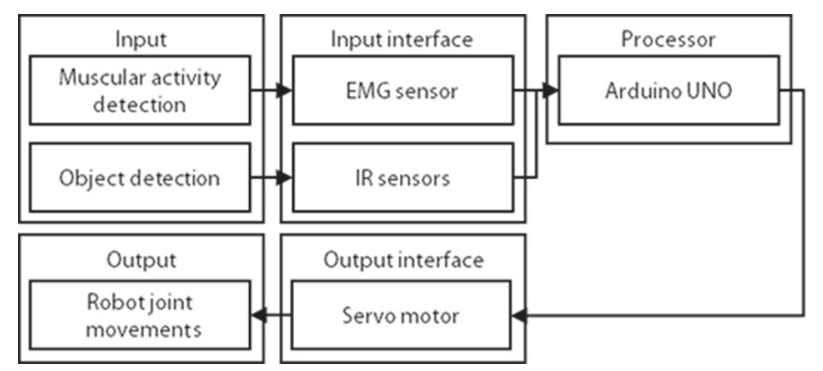

Fig. 7. A block diagram of the system.

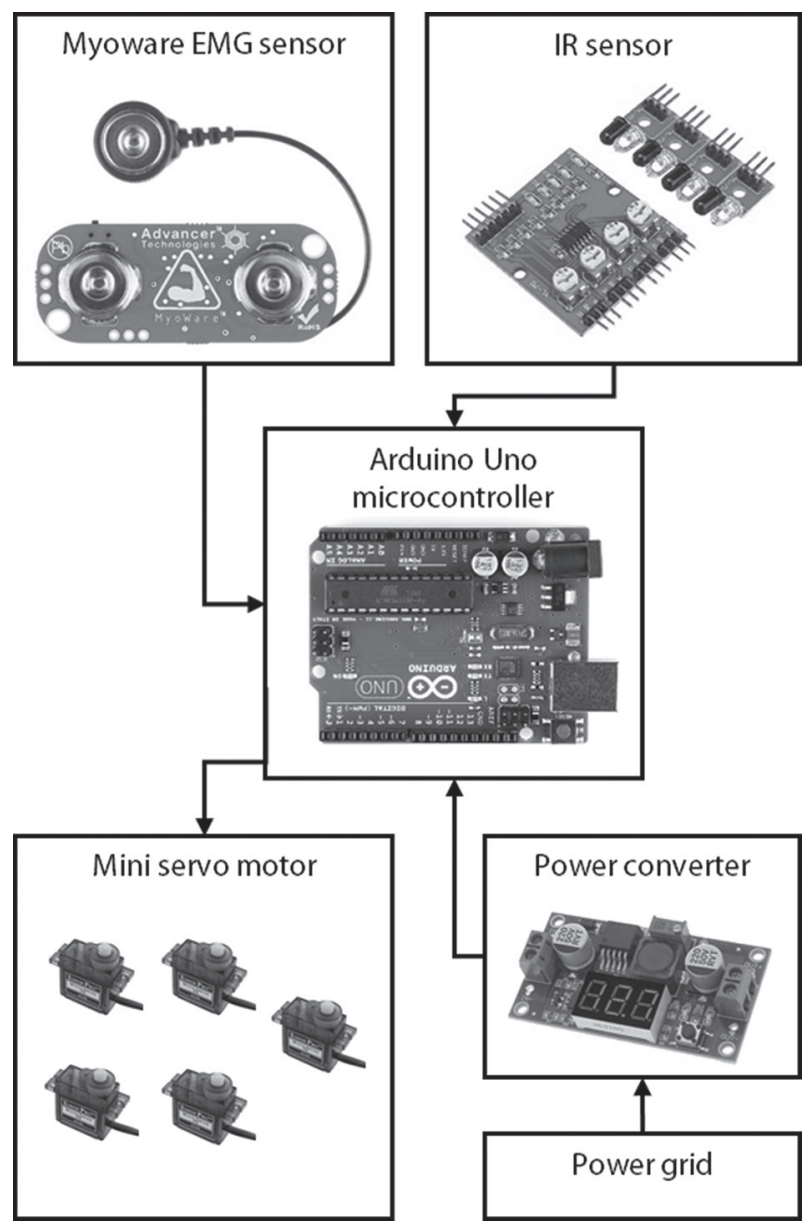

Fig. 8. Electronics hardware configuration of the system.

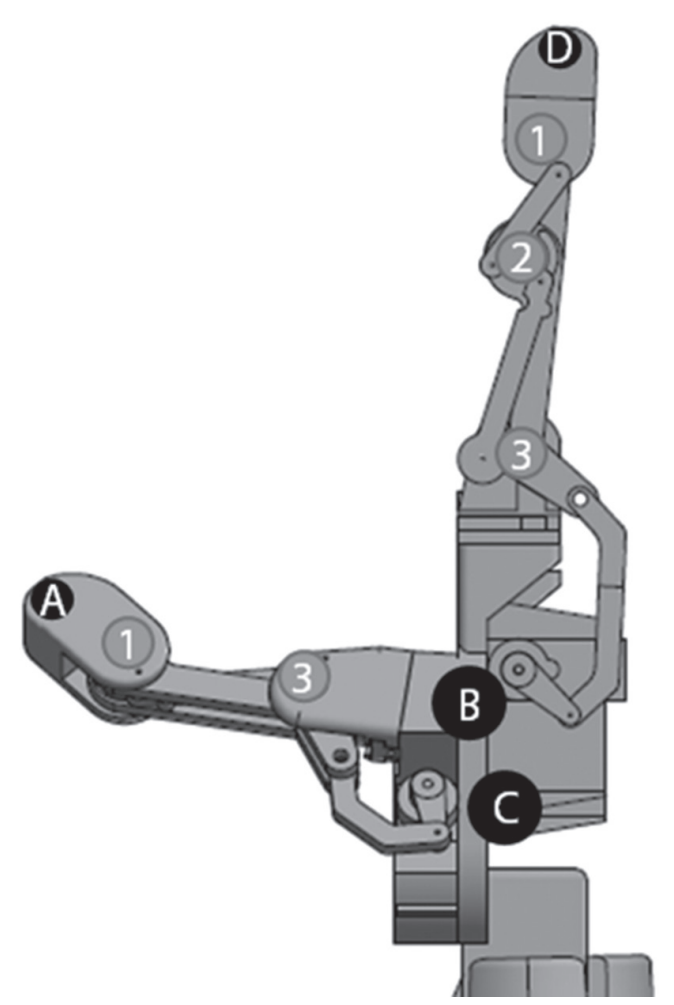

Fig. 9. Reflective marker placement setup: $1=$ distal interphalangeal, $2=$ proximal interphalangeal, $3=$ metacarpophalangeal. 


\subsection{COMPLETED PROTOTYPE}

\subsubsection{Motion analysis}

The motion capture experiment is the motion analysis of the moving part of the actual finger structure. This experiment is used to measure the maximum and minimum motion range of each joint, and the results were compared to the expected motion range, as mentioned in Table 2 above. The equipment used in this experiment were eight units of OptiTrack Flex 13 motion tracking cameras, a unit of Basler acA640-120gs synchronizing camera and nine units of reflective markers. Reflective markers are set up on the structure, as shown in Fig. 9. The robot structure acts as a subject placed in the middle of the workspace and all cameras face the subject, as shown in Fig. 10. The robot structure was controlled to perform opened hand palm and grasping motion postures for alternately 2 seconds, repeated three times for each sample. The samples were recorded five times using Venus $3 \mathrm{D}$ software with a $100 \mathrm{~Hz}$ sampling rate.

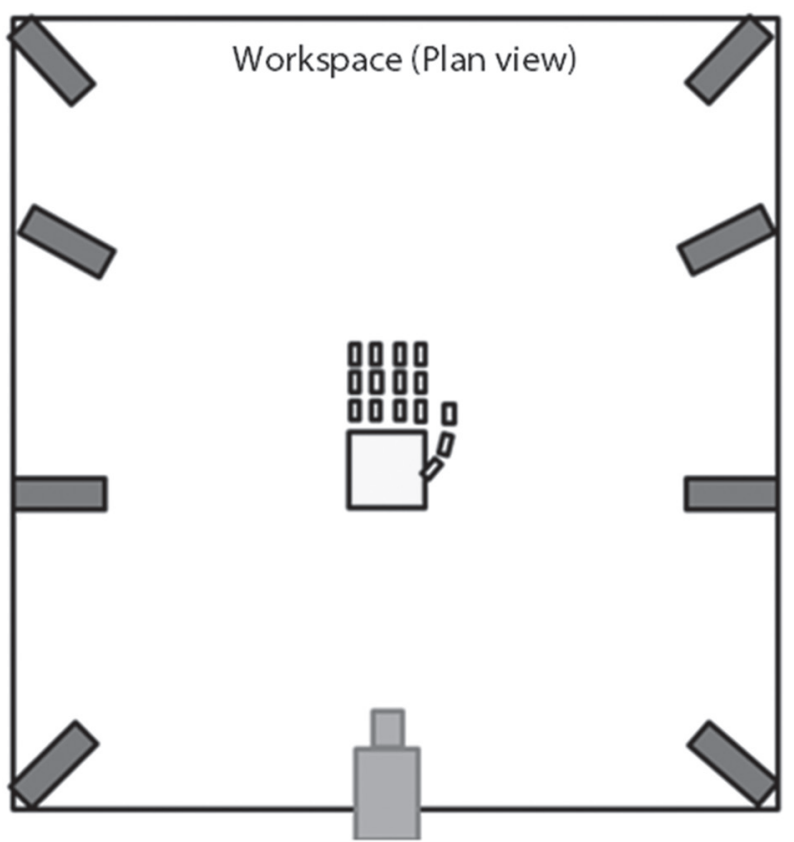

\section{Subject \\ Synchronizing camera Tracking camera}

Fig. 10. Motion capture experimental setup

\subsubsection{Electromyography (EMG) sensors placement and pre-processing analysis}

The EMG sensor was attached to the skin, where the flexor digitorum superficial (FDS) muscle is located. The FDS is the muscle used to flex the fingers, and it is suitable for muscle flexion [25]. The EMG signal was collected by using the MyoWare EMG sensor with a sampling rate of $100 \mathrm{~Hz}$. Next, the pre-processing analysis of the EMG signal was done to improve the signal quality. The analysis consists of three stages such as magnitude nor- malization, rectification, and noise filtration, as shown in Fig. 11. After that, the threshold voltage was fixed at $0.02 \mathrm{~V}$ due to less oscillation occurring at this magnitude.

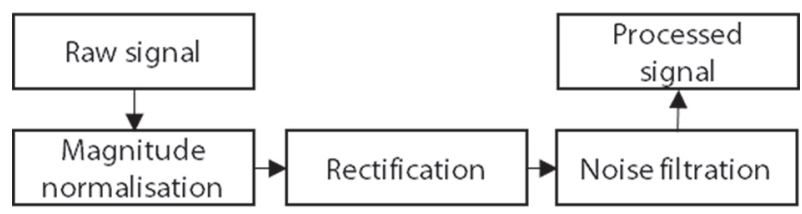

Fig. 11. Pre-processing analysis process

\subsubsection{System functionality test}

The functionality test was conducted in three stages: muscular activity detection only, object detection only, and integration of both muscular activity detection and object detection.

For the first stage, the EMG sensor is used to measure the signal generated from the targeted muscle. The signal is generated when the flexion and extension motion are done by the muscle. When the muscle flexes, the amplitude of the EMG signal is higher than usual. Thus, if the EMG signal amplitude generated more than the threshold voltage (THV) value, the robot hand would grasp the object. The robot was tested to detect the opened hand palm and grasping postures for five times alternately with $1 \mathrm{~s}$ for each posture and control robot movements in real-time. This procedure was repeated for two trials to get an accurate result.

For the second stage, it is more likely to make a variation of the robot hand posture by using the object detection method. There are four infra-red (IR) sensors used, and a sensor was allocated to each finger: index, middle, ring and baby fingers. When the IR sensor detects the object, the finger structure aligned to the sensor will be flexed. The sensors were tested for two trials by placing the obstacle on the hand palm as following motion steps, as listed in Table 3. The selected motions of the robot hand posture are commonly used in human daily life activities [26].

For the third stage, both muscular activity and object detection were integrated into one algorithm, as shown in Fig. 12. The robot system will be started with muscular activity as the first condition, and then it will proceed to object detection, as mentioned in the second stage of the functionality test. This procedure was repeated for two trials.

Table 3. Motion steps of the object detection test.

\begin{tabular}{c|cccc} 
& \multicolumn{4}{|c}{ Object Detection } \\
Step & IR1 & IR2 & IR3 & IR4 \\
$\mathbf{1}$ & On & Off & Off & Off \\
$\mathbf{2}$ & On & On & Off & Off \\
$\mathbf{3}$ & On & On & On & Off \\
$\mathbf{4}$ & On & On & On & On
\end{tabular}




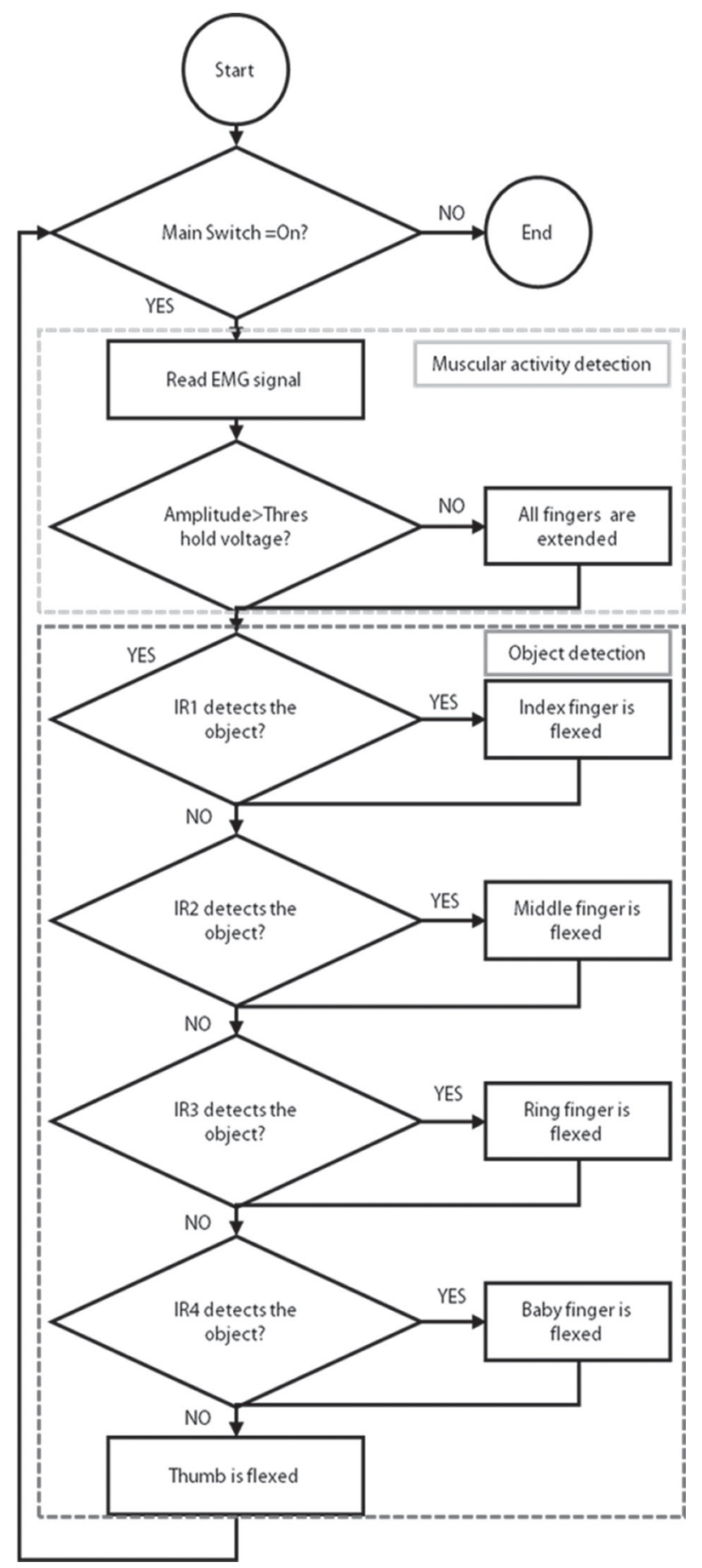

Fig. 12. Flowchart of the algorithm (Stage 3).

\section{RESULTS AND DISCUSSION}

\subsection{D PRINTED ROBOT HAND STRUCTURE AND ITS ELECTRONIC HARDWARE}

The finger structure consists of some of the 3D printed parts as body structure, and some of the steel parts such bolts and nuts as the joint connector, as shown in Fig. 13. Fig. 14 shows that the complete prototype of the robot hand is the integration of the structure and electronic hardware.

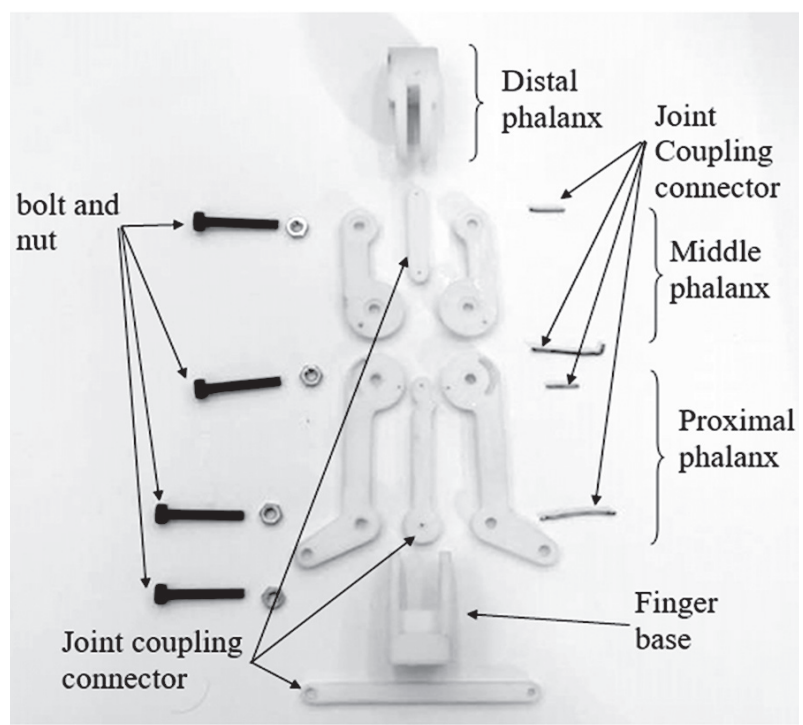

Fig. 13. Components of the finger structure disassembled

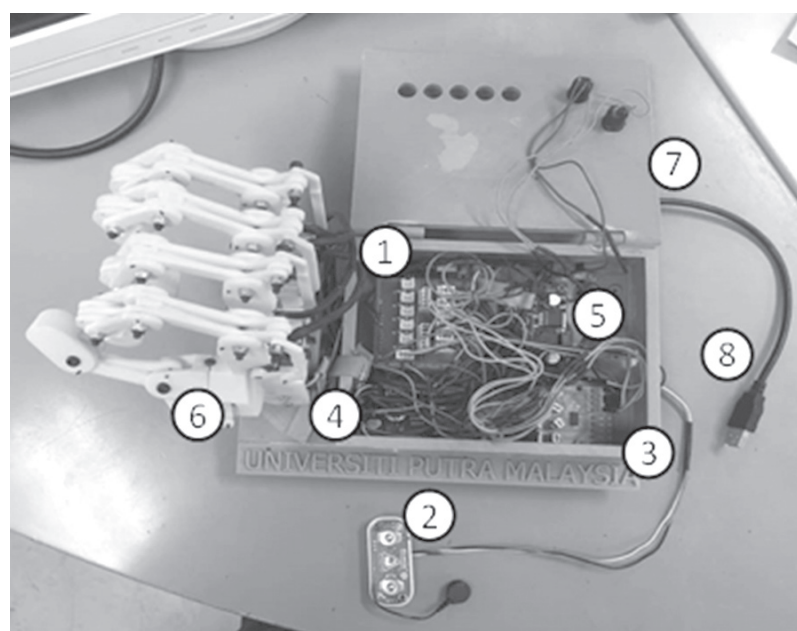

Fig. 14. Complete prototype (1) Arduino Uno board, (2) Myoware EMG sensor, (3) IR sensor, (4 \& 5) DC-DC converter, (6) robot hand structure, (7) buttons, and (8) power supply adaptor.

\subsection{MOTION ANALYSIS}

There were limitations in the experiment. Reflective markers could not be placed on all fingers, except the thumb and index finger due to insufficient space. Thus, reflective markers were placed on the index finger and the thumb only, and the index finger was assumed to be similar to the middle, ring, and baby finger due to identical structural design. Besides, the experiment was done to ensure the movement capability of the joint of the $3 \mathrm{D}$ printed structure reaching the optimum similarity to the expected motion range of structural design of the structure. By referring to Table 4, we can see that motion similarity between the 3D printed robot hand structure and structural design ranges between 72.62 and $98.43 \%$. 
Table 4. Range of motion (ROM) of the joint for structure design and actual finger

\begin{tabular}{|c|c|c|c|c|c|}
\hline \multirow{2}{*}{ Joint } & \multicolumn{5}{|c|}{ Thumb } \\
\hline & Min. & Max. & $\begin{array}{c}\text { Measured } \\
\text { ROM }\end{array}$ & $\begin{array}{c}\text { Expected } \\
\text { ROM }\end{array}$ & Similarity \\
\hline DIP & $76.1969^{\circ}$ & $159.8606^{\circ}$ & $83.6637^{\circ}$ & $85^{\circ}$ & $98.43 \%$ \\
\hline \multirow[t]{3}{*}{ MCP } & $95.2821^{\circ}$ & $172.8265^{\circ}$ & $77.5444^{\circ}$ & $90^{\circ}$ & $86.16 \%$ \\
\hline & & & Index & & \\
\hline & Min. & Max. & $\begin{array}{c}\text { Measured } \\
\text { ROM }\end{array}$ & $\begin{array}{c}\text { Expected } \\
\text { ROM }\end{array}$ & Similarity \\
\hline DIP & $90.6170^{\circ}$ & $165.0229^{\circ}$ & $74.4059^{\circ}$ & $90^{\circ}$ & $82.67 \%$ \\
\hline PIP & $101.5059^{\circ}$ & $177.7636^{\circ}$ & $76.2577^{\circ}$ & $110^{\circ}$ & $72.62 \%$ \\
\hline MCP & $99.4107^{\circ}$ & $173.351^{\circ}$ & $73.9410^{\circ}$ & $85^{\circ}$ & $86.70 \%$ \\
\hline
\end{tabular}

\subsection{ELECTROMYOGRAPHY (EMG) SIGNAL PRE-PROCESSING}

Fig. 15 shows the changes that occurred toward the EMG signal for every stage of the pre-processing analysis. The raw signal generated from the sensor is highly oscillating due to the presence of the noise signal, as shown in Fig. 15(a). After that, the signal magnitude is normalized, when the mid-value of the signal is moved to zero on the $y$-axis, as shown in Fig. 15(b). This being the case, the signal has two sides of magnitude, i.e., a positive and a negative value. Next, the signal is rectified, as shown in Fig. 15(c), so a negative magnitude of the signal is moved to the positive side of the magnitude. Then the rectified signal (red line) is smoothed as a blue line shown in Fig. 15(d) by applying the Butterworth low pass signal with $0.03 \mathrm{~Hz}$ of the cut-off frequency obtained from the magnitude spectrum graph, as shown in Fig. 16.

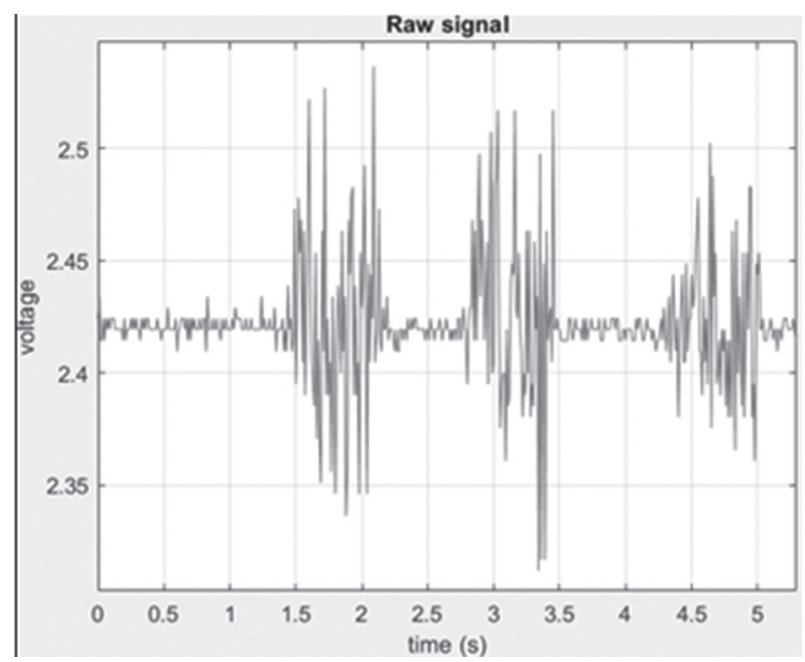

(a)

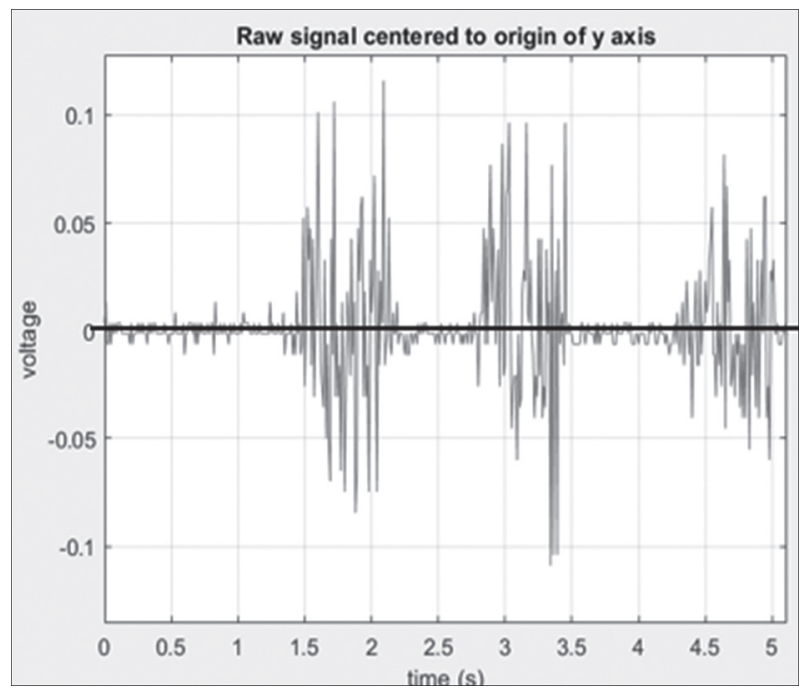

(b)

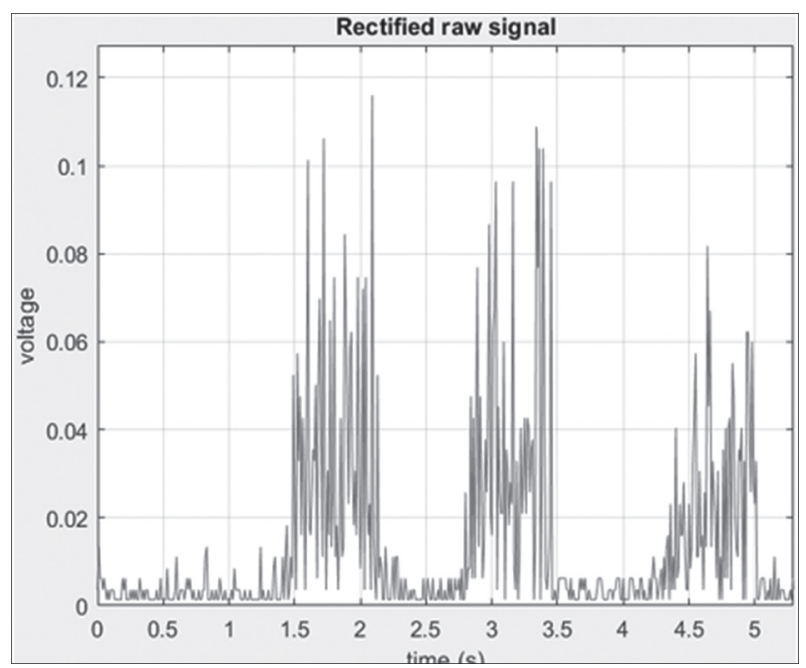

(c)

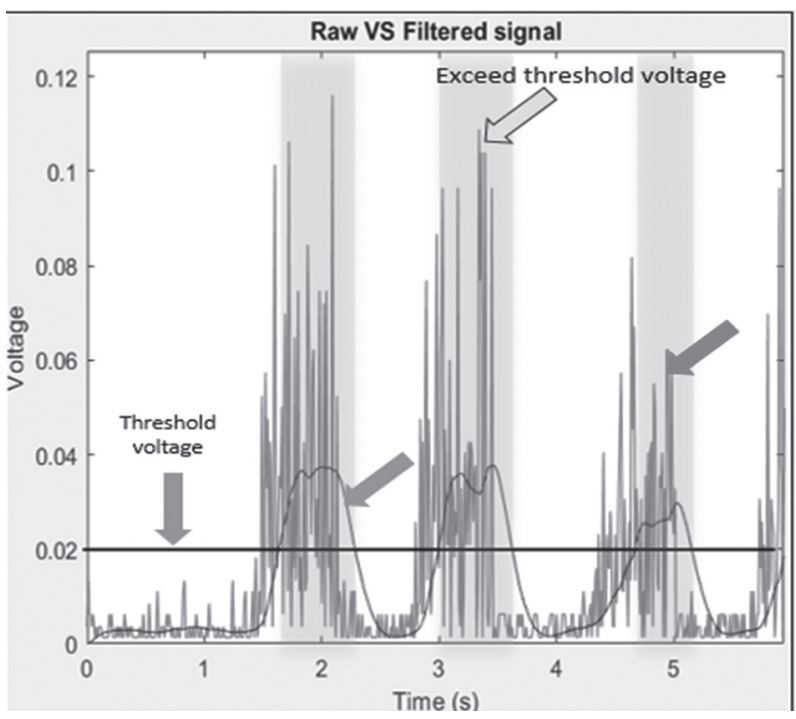

(d)

Fig. 15. Pre-processing analysis of the EMG sensor output (a) Raw signal, (b) Magnitude normalization, (c) Rectification, (d) Noise filtration. 


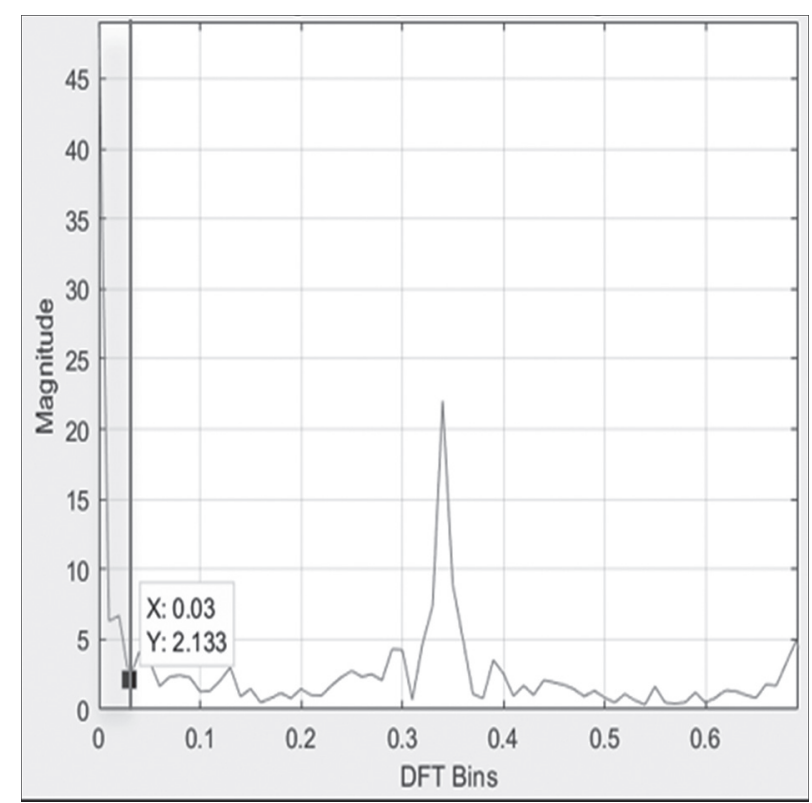

Fig. 16. The magnitude spectrum of the EMG signal.

\subsection{FUNCTIONALITY TEST}

System functionality was evaluated through some trials carrying out some tasks as mentioned in Section 2.3.3 and the result shown in Table 5. In the first stage, robot control based on the EMG sensor to perform grasping and hand palm opening was performed for five times with two trials, while the robot also succeeded to perform four different grasping postures based on object detection of the IR sensor in the second stage. In the third stage, the integration of two previous stages into one algorithm worked without any failure, as shown in Fig. 17. As a conclusion, robot control of the robot hand prototype is reliable.

Table 5. System functionality evaluation result.

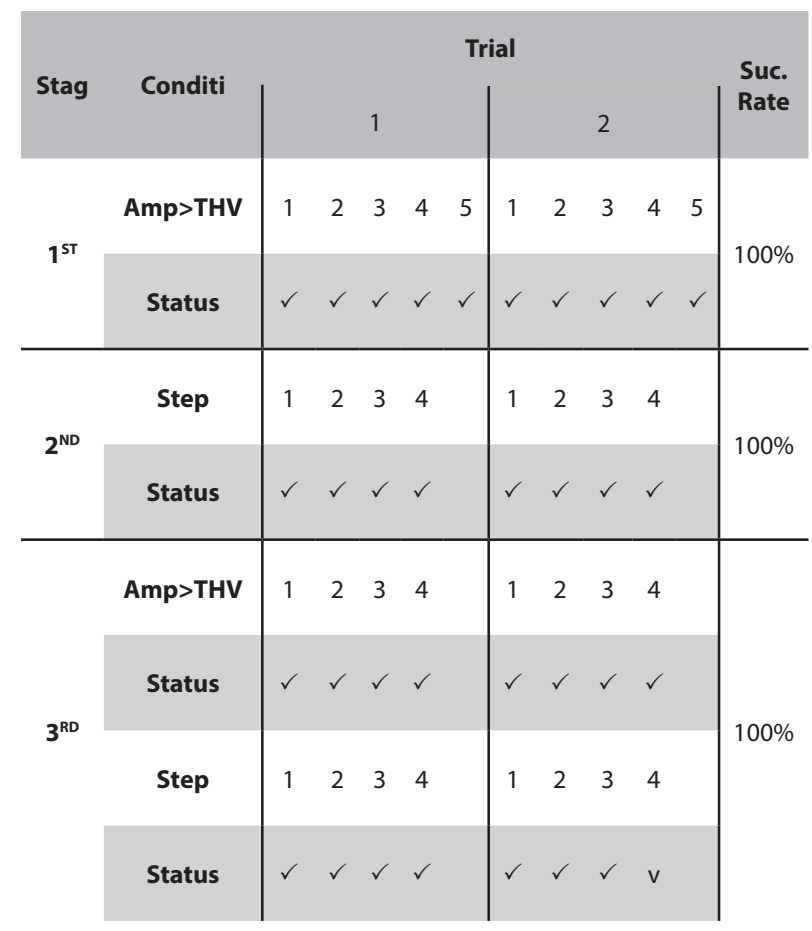

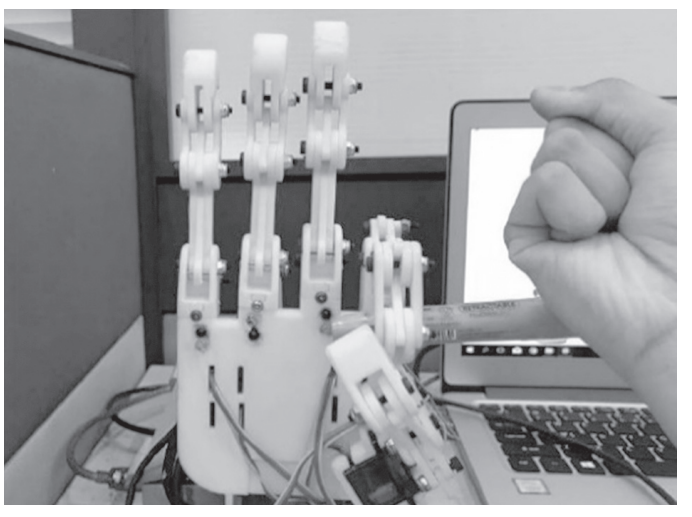

(a)

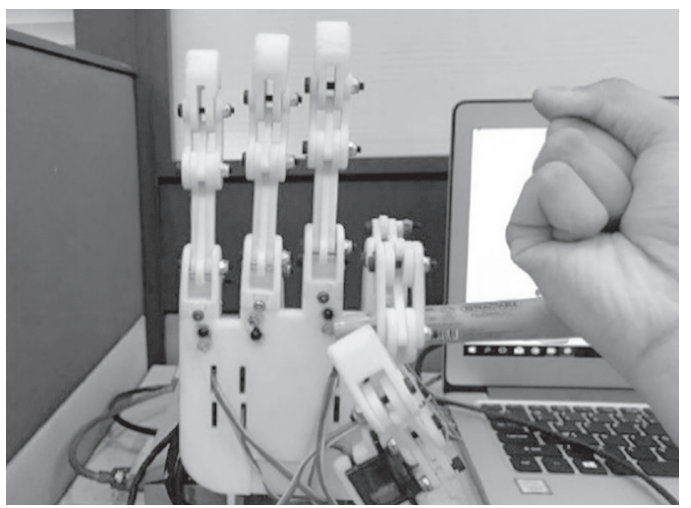

(b)

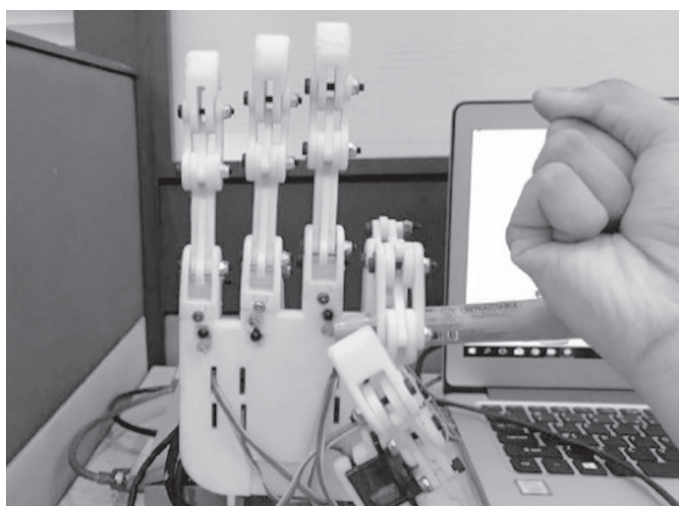

(c)

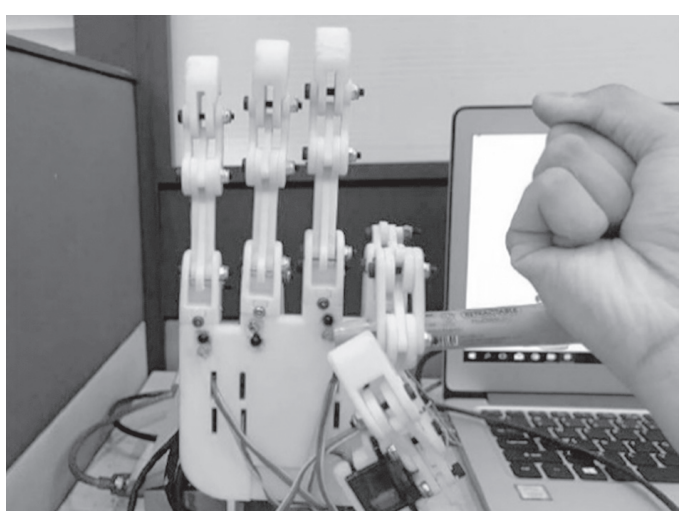

(d)

Fig. 17. Robot hand motion: (a) Step 1, (b) Step 2, (c) Step 3, and (d) Step 4. 


\section{CONCLUSION}

In conclusion, a real-time EMG controlled 3D printed robot hand prototype with improved features of structural design was developed, and its reliability was proven through the conducted functionality test.

For further study, an advanced controller using artificial intelligence could be applied to the system.

\section{REFERENCES:}

[1] Statistical Bulletin: A number of Disabled Person Registered by State and Type of Disability. Department of Social Welfare, Malaysia, 2012.

[2] H. Liu, D. Yang, S. Fan, H. Cai, "On the development of intrinsically-actuated, multisensory dexterous robotic hands", ROBOMECH Journal, Vol. 3, No. 4, 2016, pp. 1-9.

[3] K.H. Yeo, S.H.M. Ali, P.S. Menon, \& M.S. Islam "Comparison of CMOS rectifiers for micropower energy harvesters", Proceedings of the 2015 IEEE Conference on Energy Conversion (CENCON), Malaysia, 19-20 October 2015, pp. 419-423

[4] D.M. Motiur Rahaman, M.S. Islam, J. Sampe, S.H. Md. Ali, , "An architecture of ULP energy harvesting power conditioning circuit using piezoelectric transducer for wireless sensor network: A review", Asian Journal of Scientific Research, Vol. 8, No. 1, 2015, pp. 1-13.

[5] M.L.S. Mi, S.H.M. Ali, M.S. Islam, "A novel architecture of maximum power point tracking for Ultra-Low-Power based hybrid energy harvester in ubiquitous devices: A review", American Journal of Applied Sciences, Vol. 10, No. 10, 2013, pp. 1240-1251.

[6] M.R. Sarker, S.H.M. Ali, M. Othman, M.S. Islam, "Designing a low voltage energy harvesting circuits for rectified storage voltage using vibrating piezoelectric", Proceedings of the 2011 IEEE Student Conference on Research and Development (SCOReD), Cyberjaya, Malaysia, 19-20 December 2011, pp. 343-346.

[7] M.S. Bhuyan, B.Y. Majlis, M. Othman, S.H. Ali, C. Kalaivani, S. Islam, , "Development of a fluid actuated piezoelectric micro energy harvester: Finite element modeling simulation and analysis", Asian Journal of Scientific Research, Vol. 6, No. 4, 2013, pp. 691-702.
[8] M.R. Sarker, S.H.M. Ali, M. Othman, S. Islam, “Designing a battery-less piezoelectric based energy harvesting interface circuit with $300 \mathrm{mV}$ startup voltage", Journal of Physics: Conference Series, Vol. 431, No. 1, 2013.

[9] D.A. Bennett, S.A. Dalley, D. Truex, M. Goldfarb, "A Multigrasp Hand Prosthesis for Providing Precision and Conformal Grasps" IEEE/ASME Transactions on Mechatronics: a joint publication of the IEEE Industrial Electronics Society and the ASME Dynamic Systems and Control Division, Vol. 99, 2014, pp. 1-8.

[10] Y. Huang, K. Englehart, B. Hudgins, A. Chang, "A Gaussian Mixture Model Based Classification Scheme for Myoelectric Control of Powered Upper Limb Prostheses", IEEE Trans. Biomed. Eng, Vol. 52, No. 11, 2005, pp. 1801-1811.

[11] I. Timothy, 3D Printed Prosthetic Hand with Intelligent EMG Control. Bachelor's thesis, Carleton University, Canada, 10 April 2013. Available online: http://www.doe.carleton.calcourses/4th_yearprojects/Am4_Ingl is Timothy 2013.pdf-.pdf (accessed: 25 November 2018).

[12] Enabling the Future. Available online: http://enablingthefuture.org/ (accessed: 25 November 2018).

[13] P.C.S. Javier, F.O.M. Esteban, "Design and Construction of a Robot Hand Activated by Electromyographic Signals", Proceedings of the IEEE International Symposium on Robotic and Sensors Environments (ROSE2012), Magdeburg, Germany, 16-18 November 2012, pp. 2-3.

[14] S. Minsang, Y. Dukchan, K. Junghoon, C. Youngjin, "EMG-based Prosthetic Hand Control System Inspired by Missing-Hand Movement", Proceedings of the 12th International Conference on Ubiquitous Robots and Ambient Intelligence (URAI 2015), Goyang City, Korea, 28-20 October 2015, pp. 290-291.

[15] P. Sung-Woo, B. Ji-Hun, P. Jae-Han, B. Moon-Hong, "Development of an Anthropomorphic Robot Hand Aimed at Practical Use for Wide Service Robot Application", Proceedings of the 8th International Conference on Automation Science and Engineering, Seoul, South Korea, 20-12 August 2012, pp. 431-434. 
[16] C.M. Oppus, P.R.R. Prado, J.C. Escobar, J.A.G. Marinas, R.S. Reyes, "Brain-computer Interface and Voice Controlled 3D Printed Prosthetic Hand", Proceeding of the IEEE Region 10 Conference (TENCON), Marina Bay Sands, Singapore, 22-25 November 2016, pp. 2689-2692.

[17] M. Kojiro, Y. Hiroshi, “Robotics Education: Development of Cheap and Creative EMG Prosthetic Applications," Proceedings of the 2009 IEEE/RSJ International Conference on Intelligent Robots and Systems, St Louis, United States, 11-15 October 2009, pp. 2341-2346.

[18] J. Ahmed, B.M. Saiful, Y. L. Cheng, J. Roseleena, “Design and Control of a Multifingered Anthropomorphic Robotic Hand", IJMME-IJENS, Vol. 11, No. 4, 2011, pp. 24-31.

[19] F. Lotti, P. Tiezzi, G. Vassura, A. Zucchelli, "Mechanical Structures for Hands based on Compliant Mechanism Concept", Proceeding of the 7th ESA Workshop on Advanced Space Technologies for Robotics and Automation (ASTRA2002), Noordwijk, The Netherlands, 19-21 November 2002, pp. 6-14.

[20] K. Yuichi, O. Yasuhiro, I. Atsutoshi, O. Tsukasa, "Human-sized Anthropomorphic Robot Hand with Detachable Mechanism at the Wrist", Mech. And Mach. Theory, Vol. 46, No. 1, 2011, pp. 53-66.

[21] H. Huang, L. Jiang, D.W. Zhao, J.D. Zhao, “Development on a New Biomechatronic Prosthetic Hand
Based on Under-actuated Mechanism", Proceedings of the 2006 International Conference on Intelligent Robots and Systems, Beijing, China, 9-15 October 2006, pp. 3791-3793.

[22] K. Haruhisa, K. Tsuneo, U. Kazunao, K. Takashi, "Dexterous Anthropomorphic Robot hand with Distributed Tactile Sensor: Gifu Hand II", Proceedings of the IEEE International Conference on Systems, Tokyo, Japan, 12-15 October 1999, pp. 782787.

[23] Y.L. Chung, C. Almon, "The Development of a LowCost Pneumatic Air Muscle Actuated Anthropomorphic Robotic Hand.", Proceedings of the International Symposium on Robotics and Intelligent Sensors (IRIS 2012), Sarawak, Malaysia, 4-6 September 2012, pp. 737-742.

[24] K. Andrianesis, A. Tzes, “Development of Control of a Multifunction Prosthetic Hand with Shapememory Alloy Actuators", Proceedings of the Conference on Biomedical Robotics and Biomechatronics, Scottsdale, Arizona, 19-22 October 2008, pp. 517-521.

[25] Flexor Digitum Superficialis. Available online: https://rad.washington.edu/muscle-atlas/flexordigitorum-superficialis/ (accessed: 2018).

[26] M.D. Aaron, "Classifying Human Hand Use and the Activities of Daily Living", The Human Hand as an Inspiration for Robot Hand Development, Springer International Publishing, 2014, pp. 201-216 